\title{
Ultrasound longitudinal-wave anisotropy estimation in muscle tissue
}

\author{
Naiara Korta Martiartu, Saule Simutè, Thomas Frauenfelder, and Marga B. Rominger
}

\begin{abstract}
The velocity of ultrasound longitudinal waves (speed of sound) is emerging as a valuable biomarker for a wide range of diseases, including musculoskeletal disorders. Muscles are fiber-rich tissues that exhibit anisotropic behavior, meaning that velocities vary with the wave-propagation direction. Quantifying anisotropy is therefore essential to improve velocity estimates while providing a new metric that relates to both muscle composition and architecture. This work presents a method to estimate longitudinal-wave anisotropy in transversely isotropic tissues. We assume elliptical anisotropy and consider an experimental setup that includes a flat reflector located in front of the linear probe. Moreover, we consider transducers operating multistatically. This setup allows us to measure first-arrival reflection traveltimes. Unknown muscle parameters are the orientation angle of the anisotropy symmetry axis and the velocities along and across this axis. We derive analytical expressions for the relationship between traveltimes and anisotropy parameters, accounting for reflector inclinations. To analyze the structure of this nonlinear forward problem, we formulate the inversion statistically using the Bayesian framework. Solutions are probability density functions useful for quantifying uncertainties in parameter estimates. Using numerical examples, we demonstrate that all parameters can be well constrained when traveltimes from different reflector inclinations are combined. Results from a wide range of acquisition and medium properties show that uncertainties in velocity estimates are substantially lower than expected velocity differences in muscle. Thus, our formulation could provide accurate muscle anisotropy estimates in future clinical applications.

Index Terms-speed of sound, longitudinal waves, anisotropy, transverse isotropy, muscle, ultrasound, Bayesian inference, uncertainty quantification
\end{abstract}

\section{INTRODUCTION}

Speed-of-sound estimation in tissue using ultrasound has attracted considerable attention in recent years [1]-[7]. Speed of sound refers to the propagation velocity of longitudinal waves, which are typically used for image formation in ultrasound systems. This property contains clinically relevant information about tissue composition and shows great promise as a biomarker for a wide range of diseases. Clinical applications involving longitudinal-wave velocities include, for instance, breast cancer screening [1], [8], [9], hepatic steatosis assessment [10], [11], and diagnosis of musculoskeletal disorders [12], [13].

Unlike breast and liver tissue, muscles exhibit anisotropic mechanical properties due to their fibrous structure. Velocities

Naiara Korta Martiartu, Thomas Frauenfelder, and Marga B. Rominger are with the Zurich Ultrasound Research and Translation (ZURT) group, Institute of Diagnostic and Interventional Radiology, University Hospital Zürich, Zürich CH-8091, Switzerland (e-mail: naiara.kortamartiartu@usz.ch)

Saule Simute was with the Department of Earth Sciences, ETH Zurich, Zurich CH-8092, Switzerland. vary with the ultrasound wave-propagation direction, showing higher values along fiber direction than across fibers. Empirical studies in ex-vivo human and animal tissues have reported velocity differences of up to $24 \mathrm{~m} / \mathrm{s}$ [14]-[18]. Hence, failure to properly account for anisotropy can result in unreliable velocity estimates. Quantifying anisotropy is clinically interesting mainly for two reasons. On the one hand, it can provide improved velocity estimates, which are informative about muscle composition [13]. On the other hand, this property is directly related to the muscle fiber distribution, encoding also information about muscle architecture.

Anisotropy estimation can be particularly relevant for monitoring sarcopenia cost-efficiently. This is an age-related musculoskeletal disorder characterized by the progressive loss of both muscle mass and function. Ultrasound velocities are strongly correlated to reference standards for quantifying muscle mass loss [13] and have proven promising for differentiating young and older populations [12]. However, the loss in muscle mass is not correlated to the loss in muscle function [19], and both are required to assess this pathology accurately [20]. Current standards to measure muscle function, which is related to the muscle fiber arrangement [21], are based on questionnaires or tests [20]; thus, they do not include any quantitative imaging tool. In this context, anisotropy could bring significant benefits for assessing sarcopenia.

Methods to characterize the anisotropy of (quasi-)longitudinal waves are relatively unexplored in the literature. Studies addressing this topic have only focused on in-vitro measurements, where experimental setups are not appropriate for clinical examinations [14][18]. Characterization of anisotropy in shear waves, on the contrary, is an active research field. Lee et al. [22] developed an approach termed elastic tensor imaging (ETI) to map myocardial fiber directions based on shear-wave anisotropy. ETI uses either linear-probe rotations or 2D matrix-array probes [23] to measure shear-wave velocities at different propagation directions. From here, fiber orientation angles can be extracted by assuming the medium as transversely isotropic. Measurements in animal myocardial samples have demonstrated strong correlations of ETI with histological data [22] and diffusion tensor magnetic resonance imaging [24]. A similar approach using 2D matrix probes was also suggested by Wang et al. [25], who generalized the method to cases in which the shear waves excitation push is not perpendicular to fibers. Shear-wave velocity measurements, however, are prone to artifacts caused by tissue inhomogeneities. To circumvent this, Hossain et al. [26] 
proposed measuring tissue peak displacements at locations of the shear-wave excitation source. Variations of this quantity as a function of the probe orientation was seen to correlate with anisotropy in shear moduli [26]. This approach showed promising results, for example, for monitoring the status of renal transplant in humans [27].

Shear and longitudinal waves interrogate fundamentally different, but complementary, mechanical tissue properties [28]. Due to the acquisition setup, their propagation directions are perpendicular to each other; thus, shear-wave techniques cannot be directly extrapolated to longitudinal waves. The goal of this work is to propose a method to quantify the anisotropy of longitudinal waves and analyze its feasibility for clinical applications. In section II, we derive the analytical expression of the relationship between wave-propagation traveltimes and muscle anisotropy, that is, we derive our forward problem. Section III briefly introduces the Bayesian inversion approach used in this study. We then analyze the nature of the proposed problem with various numerical examples in section IV. Finally, section V summarizes key aspects of the method and carefully discusses its clinical relevance and potential improvements.

\section{TRAVELTIME MODELLING IN ANISOTROPIC MEDIA}

The alignment of fibers in muscles causes anisotropy in mechanical muscle properties. Commonly, muscle tissue is described as a transversely isotropic medium with the symmetry axis along the fiber direction [25], [26], [29], [30]. Such a medium is characterized by five independent elastic parameters, describing, for instance, the longitudinal- and shear-wave velocities along and across the symmetry axis. In soft tissue, however, shear-wave velocities are negligible in comparison to longitudinal-wave velocities [31]. Therefore, it is possible to describe muscle properties using only three independent parameters. In this study, we assume elliptical anisotropy, which is a special case of transverse isotropy. The validity of this assumption is discussed in Appendix A. The three independent parameters are then the orientation angle $\varphi$ of the anisotropy symmetry axis and the longitudinal-wave velocities along $\left(v_{1}\right)$ and across $\left(v_{2}\right)$ this axis. The group (ray) velocity $v(\theta)$ in an arbitrary propagation direction $\theta$ satisfies [32]

$$
\frac{v^{2}(\theta)}{v_{1}^{2}} \sin ^{2}(\theta-\varphi)+\frac{v^{2}(\theta)}{v_{2}^{2}} \cos ^{2}(\theta-\varphi)=1,
$$

where the angles $\theta$ and $\varphi$ are illustrated in Fig.1(a).

Traveltimes of different arrivals are affected by the direction-dependent velocity $v(\theta)$, and we can use them to retrieve tissue anisotropy parameters $\mathbf{m}=\left(v_{1}, v_{2}, \varphi\right)$. For simplicity, we consider the muscle as a two-dimensional homogeneous medium. Using (1) and trigonometric identities, we find that the traveltime $t_{\mathrm{AB}}$ between positions $\mathrm{x}_{\mathrm{A}}$ and $\mathrm{x}_{\mathrm{B}}$
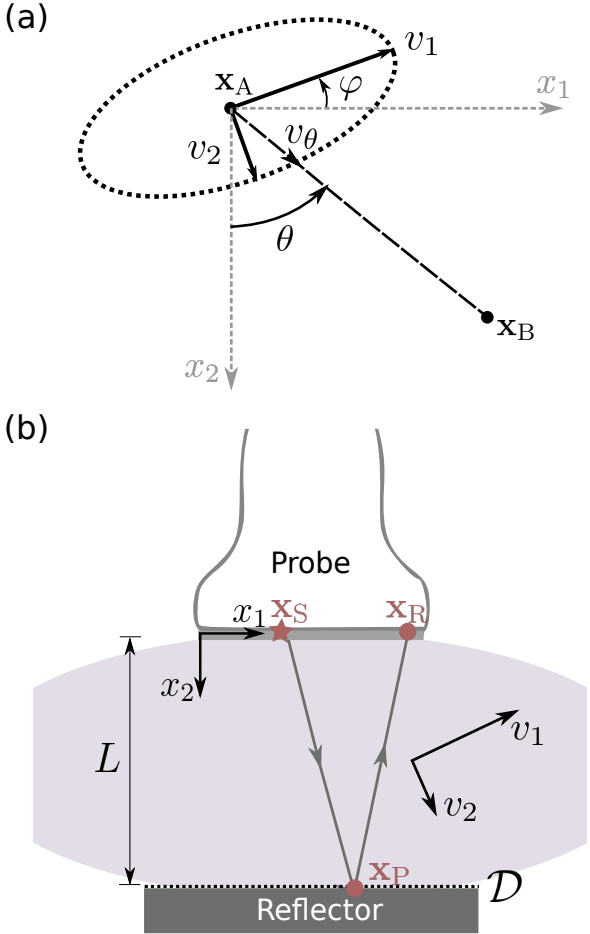

Fig. 1. Schematic representation of the anisotropic medium and experimental setup considered in this study. (a) Wavefronts in elliptical anisotropic media are ellipsoidal. Parameters $v_{1}$ and $v_{2}$ represent velocities along and across muscle fibers, and $\varphi$ describes the orientation of fibers with respect to the coordinate system. In an arbitrary propagation direction $\theta$ connecting $\mathbf{x}_{\mathrm{A}}$ and $\mathbf{x}_{\mathrm{B}}$, waves propagate with velocity $v_{\theta}=v(\theta)$. (b) Our experimental setup includes a flat reflector in front of the probe, with tissue in between. The probe-reflector distance $L$ is controlled by a sensor. We measure first-arrival reflection traveltimes of ultrasound signals emitted from $\mathbf{x}_{\mathrm{S}}$ and received at $\mathbf{x}_{\mathrm{R}}$, with $\mathbf{x}_{\mathrm{P}} \in \mathcal{D}$ indicating the reflection point.

is given by

$$
\begin{aligned}
t_{\mathrm{AB}}^{2}= & \frac{1}{v_{1}^{2}}\left[\left(x_{1, \mathrm{~B}}-x_{1, \mathrm{~A}}\right) \cos \varphi-\left(x_{2, \mathrm{~B}}-x_{2, \mathrm{~A}}\right) \sin \varphi\right]^{2}+ \\
& \frac{1}{v_{2}^{2}}\left[\left(x_{1, \mathrm{~B}}-x_{1, \mathrm{~A}}\right) \sin \varphi+\left(x_{2, \mathrm{~B}}-x_{2, \mathrm{~A}}\right) \cos \varphi\right]^{2} .
\end{aligned}
$$

From this equation we observe that $t_{\mathrm{AB}}$ is nonlinearly related to anisotropic parameters $\mathbf{m}$. In the special situation where the orientation of the symmetry axis is known, we obtain a linear relationship between squared traveltimes $t^{2}$ and squared slownesses $1 / v_{1}^{2}$ and $1 / v_{2}^{2}$.

\section{A. Reflector-based experimental setup}

In this study, we consider an experimental setup that includes a reflector located opposite to the linear ultrasound probe [see Fig. 1(b)], with the probe-reflector distance $L$ controlled by a distance sensor [4]. This setup has already been applied in various clinical studies for the assessment of breast [33], [34] and muscle tissue [12], [13], [31], [35]. The reflector allows us to measure the first-arrival reflection traveltimes $t_{\mathrm{SR}}$ of waves propagating from a source at $\mathbf{x}_{\mathrm{S}}$ to 
a receiver at $\mathrm{x}_{\mathrm{R}}$. These traveltimes can be expressed using Fermat's principle as

$$
\min _{\mathbf{x}_{\mathrm{P}} \in \mathcal{D}} t_{\mathrm{SR}}\left(\mathbf{x}_{\mathrm{P}}\right), \quad \text { where } \quad t_{\mathrm{SR}}\left(\mathbf{x}_{\mathrm{P}}\right)=t_{\mathrm{SP}}\left(\mathbf{x}_{\mathrm{P}}\right)+t_{\mathrm{PR}}\left(\mathbf{x}_{\mathrm{P}}\right),
$$

where $\mathcal{D}$ refers to the set of points $\mathrm{x}_{\mathrm{P}}$ at the reflector-tissue interface [see Fig. 1(b)], and traveltimes of each path are computed using (2).

Unlike in isotropic media, the reflection point $\mathrm{x}_{\mathrm{P}}^{\min }$ for the minimum traveltime does not necessarily lie on the mid-point between $\mathbf{x}_{S}$ and $\mathbf{x}_{\mathrm{R}}$ in anisotropic media. To find an analytical solution to (3), we place the origin of the coordinate system as shown in Fig. 1(b) and assume that the location of the reflection point satisfies $\mathbf{x}_{\mathrm{P}}^{\min }=\left(\left(x_{1, \mathrm{~S}}+x_{1, \mathrm{R}}\right) / 2+\delta, L\right)$, where $\delta$ is a constant value. That is, we assume that $\mathbf{x}_{\mathrm{P}}^{\min }$ is shifted from the source-receiver mid-point position by the same constant $\delta$ for every source-receiver combination. To find the value of $\delta$, we consider, for simplicity, the zero-offset case in which $\mathrm{x}_{\mathrm{S}}=\mathrm{x}_{\mathrm{R}}$, and we solve (3) using

$$
\frac{d t_{\mathrm{SR}}}{d x_{\mathrm{P}}}=2 \frac{d t_{\mathrm{SP}}}{d \delta}=0 .
$$

The reflection point is then

$$
\mathbf{x}_{\mathrm{P}}^{\min }=\left(\frac{x_{1, \mathrm{~S}}+x_{1, \mathrm{R}}}{2}+\frac{L \sin 2 \varphi\left(v_{2}^{2}-v_{1}^{2}\right)}{2\left(v_{1}^{2} \sin ^{2} \varphi+v_{2}^{2} \cos ^{2} \varphi\right)}, L\right) .
$$

The equation (5) shows that the reflection point is located at the source-receiver midpoint only when the medium is isotropic $\left(v_{1}=v_{2}\right)$ or the anisotropy symmetry axis is aligned with our coordinate system $(\varphi=0)$. For muscle tissue, we expect $v_{1}>v_{2}$ for $\varphi \in[-\pi / 4, \pi / 4)$, i.e., waves propagating faster along than across fiber direction [14]. Therefore, $\delta$ can be either positive or negative depending on the sign of $\varphi$.

Upon inserting (5) in (3) and (2), it is possible to see that the path with the minimum traveltime satisfies $t_{\mathrm{SP}}\left(\mathrm{x}_{\mathrm{P}}^{\min }\right)=$ $t_{\mathrm{RP}}\left(\mathrm{x}_{\mathrm{P}}^{\mathrm{min}}\right)$. The fastest ray path is therefore the one with equal traveltime along each segment. This also means that the mirror image of the receiver, namely a virtual equivalent receiver R' below the reflector, is located at $x_{R^{\prime}}=2 x_{P}$. The first-arrival reflection traveltime between $x_{S}$ and $x_{R}$ is then

$$
t_{\mathrm{SR}}^{2}\left(\mathbf{x}_{\mathrm{P}}^{\min }\right)=\frac{d^{2}}{v^{2}(\theta=\pi / 2)}+\frac{4 L^{2} v^{2}(\theta=\pi / 2)}{v_{1}^{2} v_{2}^{2}},
$$

with $v^{2}(\theta=\pi / 2)$ given by (1) and $d=x_{1, \mathrm{R}}-x_{1, \mathrm{~S}}$ being the source-receiver offset. This equation establishes the relationship between observations $t_{\mathrm{SR}}$ and unknown muscle properties $\mathbf{m}=\left(v_{1}, v_{2}, \varphi\right)$. Thus, the forward problem considered in this study is nonlinear. When the anisotropy symmetry axis is aligned with the coordinate system $(\varphi=0),(6)$ reduces to

$$
t_{\mathrm{SR}}^{2}\left(\mathbf{x}_{\mathrm{P}}^{\min }\right)=\frac{d^{2}}{v_{1}^{2}}+\frac{4 L^{2}}{v_{2}^{2}}
$$

and, as previously observed, $t_{\mathrm{SR}}^{2}$ becomes linearly related to squared slownesses $1 / v_{1}^{2}$ and $1 / v_{2}^{2}$.

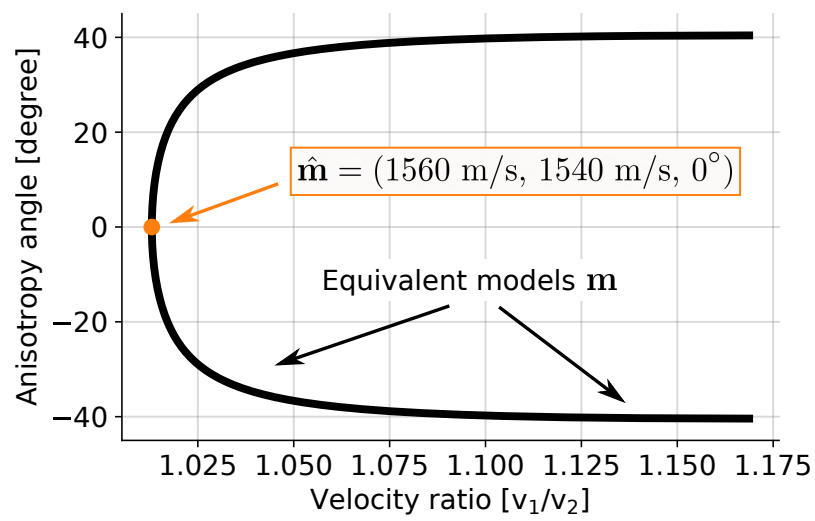

Fig. 2. Muscle models satisfying the conditions (8) and, thus, providing equal traveltimes. For this example, we take the reference model $\hat{\mathbf{m}}=\left(1560 \mathrm{~m} / \mathrm{s}, 1540 \mathrm{~m} / \mathrm{s}, 0^{\circ}\right)$ and represent equivalent models $\mathbf{m}$ for $\varphi \in\left[-45^{\circ}, 45^{\circ}\right)$. Because muscle models are defined by three parameters, we represent the anisotropy angle $\varphi$ versus the velocity ratio $v_{1} / v_{2}$ for visualization.

\section{B. Non-uniqueness}

In this section, we demonstrate that traveltimes satisfying (6) are not sufficient to constrain muscle properties uniquely. For brevity, we omit the dependency on $\mathrm{x}_{\mathrm{P}}^{\min }$.

Let us assume that the traveltimes $t_{\mathrm{SR}}^{2}(\hat{\mathbf{m}})$ are obtained from muscle properties $\hat{\mathbf{m}}$. If $t_{\mathrm{SR}}^{2}(\hat{\mathbf{m}})$ is uniquely defined by $\hat{\mathbf{m}}$, then any other $\mathbf{m}$ giving the same traveltimes $t_{\mathrm{SR}}^{2}(\hat{\mathbf{m}})=$ $t_{\mathrm{SR}}^{2}(\mathbf{m})$ must satisfy $\hat{\mathbf{m}}=\mathbf{m}$. For simplicity, we take $\hat{\mathbf{m}}=\left(\hat{v}_{1}, \hat{v}_{2}, \hat{\varphi}=0^{\circ}\right)$ and $\mathbf{m}=\left(v_{1}, v_{2}, \varphi\right)$ and consider a single source-receiver pair. Equating (6) and (7), we see that both muscle parameters give the same traveltimes when conditions

$$
\begin{gathered}
\hat{v}_{1} \hat{v}_{2}=v_{1} v_{2} \\
v_{1}^{2} \sin ^{2} \varphi+v_{2}^{2} \cos ^{2} \varphi=\hat{v}_{2}^{2}
\end{gathered}
$$

are satisfied. Therefore, we can always find different muscle models giving same traveltime observations, even when we exclude the intrinsic periodicity of $\varphi$ (i.e., $\mathbf{m}(\varphi)=\mathbf{m}(\varphi+2 \pi))$ and the obvious symmetry of the elliptical anisotropy $\left(v_{1} \rightarrow v_{2}\right.$ when $\varphi \rightarrow \varphi+\pi / 2$ ). A concrete example of all equivalent muscle models (in terms of traveltimes) is shown in Fig. 2. The figure shows that $\varphi$ is not constrained by the forward problem in (6). Hence, we require additional types of observations. Note that including multiple sources in the previous example could not constrain the problem because the conditions (8) do not depend on source and receiver locations.

\section{Reflector inclination: sources of uncertainties as new con- straints}

The simplest way to constrain the anisotropy angle is by combining data acquired from multiple muscle sides. This is equivalent to rotating the tissue with respect to the probe location. For in-vivo studies, however, we can only access the muscle from a single side of the anisotropy plane. To circumvent this limitation, we suggest taking advantage of the 
reflector inclination, which is unavoidable in clinical practice and regarded as a source of uncertainties. The reflector inclination will generate ray paths with orientations that are different from our previous setup. Therefore, we suggest combining data from multiple inclination angles to constrain muscle anisotropy. In the following, we assume that the inclination angle is controlled using, for instance, B-mode images, and we derive the corresponding forward problem.

Let us denote $\alpha$ the reflector inclination angle with respect to the $x_{1}$-axis. We can use equations derived above by rotating the whole setup in order to align the reflector with the $x_{1}$-axis. In this situation, the anisotropy angle becomes $\varphi \rightarrow \varphi+\alpha$, the probe is inclined by $\alpha$ with respect to the $x_{1}$-axis, and the probe-reflector distance becomes $L \rightarrow L \cos \alpha$. The probereflector distance is measured from the origin of the coordinate system, which is located in the first transducer element of the probe [see Fig. 1(b)]. Using geometrical identities and the previous result in (5), the reflection point $\mathrm{x}_{\mathrm{P}}^{\min }=\left(x_{1, \mathrm{P}}^{\min }, L \cos \alpha\right)$ becomes

$$
x_{1, \mathrm{P}}^{\min }=x_{1, \mathrm{~S}}+\frac{d \cos \alpha}{2}-\frac{d^{2} \sin 2 \alpha / 2+\delta^{\prime} d \sin \alpha}{2 L^{\prime} \cos \alpha+d \sin \alpha}+\delta^{\prime},
$$

with

$$
\delta^{\prime}=\frac{\left(L^{\prime} \cos \alpha+d \sin \alpha\right) \sin 2 \varphi\left(v_{2}^{2}-v_{1}^{2}\right)}{2\left(v_{1}^{2} \sin ^{2}(\varphi+\alpha)+v_{2}^{2} \cos ^{2}(\varphi+\alpha)\right)}
$$

and

$$
L^{\prime}=L+x_{1, \mathrm{~S}} \sin \alpha
$$

As before, we replace $x_{P}^{\min }$ in (3) and (2) to find the total traveltime

$$
t_{\mathrm{SR}}^{2}=\frac{d^{2}}{v^{2}(\pi / 2)}+\frac{\left(4 L^{\prime 2} \cos ^{2} \alpha+2 L^{\prime} d \sin 2 \alpha\right)}{v_{1}^{2} \sin ^{2}(\varphi+\alpha)+v_{2}^{2} \cos ^{2}(\varphi+\alpha)} .
$$

This equation is the generalization of (6), which we obtain when $\alpha=0$.

\section{Validation with numerical simulations}

To validate our traveltime modelling, we use numerical wave propagation simulations. We model the wave propagation in muscle using the two-dimensional time-domain elastic wave equation with shear modulus equal to zero, i.e.,

$$
\rho \partial_{t}^{2} u(\mathbf{x}, t)-\nabla \cdot(\mathbf{D} \nabla u(\mathbf{x}, t))=f\left(\mathbf{x}_{\mathbf{S}}, t\right) .
$$

Here, $f$ is the external source generated from $\mathbf{x}_{\mathbf{S}}, u$ is the scalar displacement potential, $\rho$ is the muscle density, and $\mathbf{D}$ is a second-order symmetric positive tensor describing the direction-dependent velocities $v$. If the anisotropy is aligned with the coordinate system, $\mathbf{D}$ is a diagonal matrix with elements $D_{11}=\rho v_{1}^{2}$ and $D_{22}=\rho v_{2}^{2}$. For tilted anisotropy, we apply the rotation matrix to derive the elements of $\mathbf{D}$. We assume muscle density as $\rho=1000 \mathrm{~kg} / \mathrm{m}^{2}$. Numerical simulations are computed using the spectral-element solver Salvus [36].

Fig. 3(a) compares traveltimes measured from wave propagation simulations with those modelled using (12). For this example, we use the muscle model $\mathbf{m}=\left(1560 \mathrm{~m} / \mathrm{s}, 1540 \mathrm{~m} / \mathrm{s}, 15^{\circ}\right)$, the reflector inclination $\alpha=5^{\circ}$, a probe-reflector distance of $L=6 \mathrm{~cm}$, and a probe of $4 \mathrm{~cm}$ length with 128 transducer elements. We use the first transducer element as a source with a Ricker wavelet of $2 \mathrm{MHz}$ center frequency and all transducers as receivers. Our approach predicts traveltimes accurately, even though the frequencies of simulated ultrasonic waves are lower than those used commercially $(5-12 \mathrm{MHz})$. The traveltime modelling presented here is based on the ray theory, which assumes infinite frequencies. The higher the frequencies, the more accurate our approximation is, as demonstrated in Fig. 3(b). This result also shows that the method is still valid for relatively low frequencies, but it fails to correctly predict traveltimes at very low frequencies $(<0.25 \mathrm{MHz})$ due to finite-frequency effects [6].

(a)
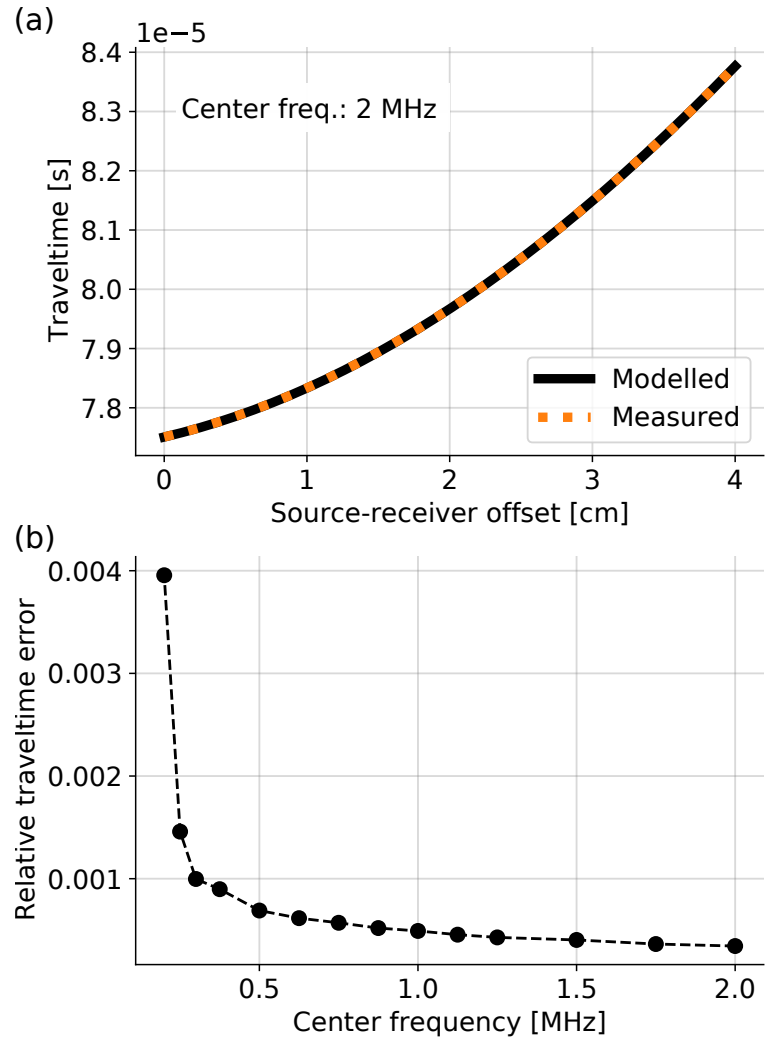

Fig. 3. Forward problem validation. (a) Comparison of traveltimes measured from numerical wave propagation simulations with those modelled using (12). Traveltimes are represented with respect to the source-receiver offset. (b) Relative error between measured and modelled traveltimes as function of the center frequency of the emitted signal. Relative errors are computed with the Euclidean norm. The accuracy of our forward problem increases with the frequency. The same setup and medium is used in both figures.

\section{STATISTICAL INVERSE PROBLEM}

Estimating muscle anisotropic properties $\mathbf{m}$ from traveltime observations $\mathbf{d}_{\mathrm{obs}}$ involves solving a nonlinear inverse problem. In principle, we can formulate this as a gradient-based optimization problem to search for the model $\mathbf{m}$ that minimizes the misfit between observed and predicted traveltimes [37]. Such deterministic approaches, however, cannot guarantee that the solution corresponds to the global minimum of the 
nonlinear function we try to minimize. They are also incapable of accurately estimating uncertainties in the solution caused by measurement noise, limited data coverage, and inaccurate forward modelling [38]. In this study, our goal is to analyze the feasibility of estimating the longitudinal-wave anisotropy from traveltime observations. For this analysis, quantifying uncertainties is crucial. Here we address the inversion statistically using the Bayesian framework. The solution is a posterior probability density function (pdf) $\pi_{\text {post }}\left(\mathbf{m} \mid \mathbf{d}_{\mathrm{obs}}\right)$ that contains the complete statistical description of model parameters [37], [38].

According to Bayes' theorem [39], [40], the posterior pdf satisfies

$$
\pi_{\text {post }}\left(\mathbf{m} \mid \mathbf{d}_{\text {obs }}\right)=k \pi_{\text {prior }}(\mathbf{m}) \pi_{\text {like }}\left(\mathbf{d}_{\text {obs }} \mid \mathbf{m}\right),
$$

where $k$ is an appropriate normalization constant, $\pi_{\text {prior }}(\mathbf{m})$ encodes our prior information on $\mathbf{m}$, and the data likelihood $\pi_{\text {like }}\left(\mathbf{d}_{\text {obs }} \mid \mathbf{m}\right)$ is the conditional probability of having observations $\mathbf{d}_{\mathrm{obs}}$ given the model $\mathbf{m}$. We can express the data likelihood explicitly as

$$
\pi_{\text {like }}\left(\mathbf{d}_{\text {obs }} \mid \mathbf{m}\right) \propto \exp \left[-\frac{1}{2}\left(\mathbf{d}-\mathbf{d}_{\mathrm{obs}}\right)^{\mathrm{T}} \boldsymbol{\Gamma}_{\mathrm{n}}^{-1}\left(\mathbf{d}-\mathbf{d}_{\mathrm{obs}}\right)\right],
$$

where $\mathbf{d}=\mathbf{F}(\mathbf{m})$ is the forward problem in (12), and $\boldsymbol{\Gamma}_{\mathrm{n}}$ is the noise covariance matrix describing uncertainties in observations. That is, $\pi_{\text {like }}\left(\mathbf{d}_{\mathrm{obs}} \mid \mathbf{m}\right)$ is a measure of the similarity between $\mathbf{d}$ and $\mathbf{d}_{\mathrm{obs}}$.

In principle, the prior $\pi_{\text {prior }}(\mathbf{m})$ can take any form. We generally express the prior in terms of individual model parameters $m_{i}$ as

$$
\pi_{\text {prior }}(\mathbf{m})=\prod_{i=1}^{N} \pi_{\text {prior }}\left(m_{i}\right)
$$

where $N$ is the number of parameters in $\mathbf{m}$. In this study, we use either a uniform distribution between a fixed range of values, i.e.,

$$
\pi_{\text {prior }}\left(m_{i}\right)= \begin{cases}\frac{1}{m_{i}^{\max }-m_{i}^{\min }}, & \text { if } m_{i} \in\left[m_{i}^{\min }, m_{i}^{\max }\right] \\ 0, & \text { otherwise }\end{cases}
$$

or a Gaussian distribution

$$
\pi_{\text {prior }}\left(m_{i}\right)=\frac{1}{\sqrt{2 \pi} \sigma_{i}} \exp \left[-\frac{\left(m_{i}-m_{i}^{0}\right)^{2}}{2 \sigma_{i}^{2}}\right],
$$

with mean $m_{i}^{0}$ and standard deviation $\sigma_{i}$.

The posterior allows us to extract useful statistical information about muscle anisotropic parameters. For instance, we can compute the probability of $\mathbf{m}$ satisfying certain conditions $\mathcal{M}_{1}$ of clinical interest as $P\left(\mathbf{m} \in \mathcal{M}_{1}\right)=\int_{\mathcal{M}_{1}} \pi_{\text {post }}\left(\mathbf{m} \mid \mathbf{d}_{\text {obs }}\right) \mathrm{d} \mathbf{m}$. This probability can be relevant in clinical decision-making when disease-related thresholds exist for anisotropic parameters. Other statistical quantities such as the expectation or marginal pdfs are also computed via similar integrals.

Unless the forward problem is linear, and the prior and noise are Gaussian, analytical expressions of the posterior are not available [37], [41]. Still, it is possible to approximate the statistical information contained in the posterior using efficient sampling techniques. In this study, we employ the Metropolis-Hastings Markov chain Monte Carlo (MCMC) algorithm [42]-[44]. The algorithm generates an ensemble of random samples of the posterior with sampling density proportional to $\pi_{\text {post }}\left(\mathbf{m} \mid \mathbf{d}_{\mathrm{obs}}\right)$. Thus, we can use this ensemble to approximate integrals related to our statistical quantities of interest.

\section{NUMERICAL EXAMPLES}

In this section, we show numerical examples illustrating the nature of the anisotropy estimation problem. Our objectives are threefold: (i) show the role of the reflector inclination in constraining the anisotropy angle, (ii) investigate the robustness of the problem under uncertain inclination angles and measurement noise, and (iii) understand the impact of the experimental setup and medium properties on solution uncertainties.

All examples shown here consider a uniform prior for velocities and anisotropy angle within the range of $[1300 \mathrm{~m} / \mathrm{s}, 1800 \mathrm{~m} / \mathrm{s}]$ and $\left[-45^{\circ}, 45^{\circ}\right)$, respectively. Moreover, we assume Gaussian observational errors with a standard deviation of $1 \%$ of the maximum traveltime values. To ensure convergence and correctly interpret the statistical results, we explore the posterior with a relatively large number of random samples, $\mathcal{O}\left(10^{7}\right)$, although fewer samples could suffice for practical purposes.

\section{A. Unconstrained problem}

In this example, we solve the Bayesian anisotropy inference using the forward problem in (6). Our goal is to illustrate how the non-uniqueness of the forward problem is mapped into the posterior. We consider the same example as in Fig. 2, where the true model is $\mathbf{m}_{\text {true }}=\left(1560 \mathrm{~m} / \mathrm{s}, 1540 \mathrm{~m} / \mathrm{s}, 0^{\circ}\right)$ and the probe-reflector distance is $L=10 \mathrm{~cm}$. We use an ultrasound probe of $4 \mathrm{~cm}$ length with 128 transducer elements from which one acts as a source, and all are in receiving mode. Our artificial observations of traveltimes are numerically computed from (6). Fig. 4 shows the solution of the inverse problem, namely the posterior pdf. Models with maximum posterior probability densities are same as those theoretically predicted in Fig. 2 and predict the observations equally likely. The width of the region with maximum probability density is related to the Gaussian noise in the data likelihood. Note that including multiple sources do not improve the nonuniqueness because the conditions (8) are independent of source and receiver locations. Unless our prior information on model parameters is stronger than a uniform distribution, the posterior will show the exact same non-uniqueness of the forward problem. However, a stronger prior would dominate the solution. For instance, a Gaussian prior would produce a maximum a posteriori point at the same location of the prior's maximum, which may not represent the true model. Hence, one should carefully interpret the posterior when the data is not informative enough on model parameters. Alternatively, 


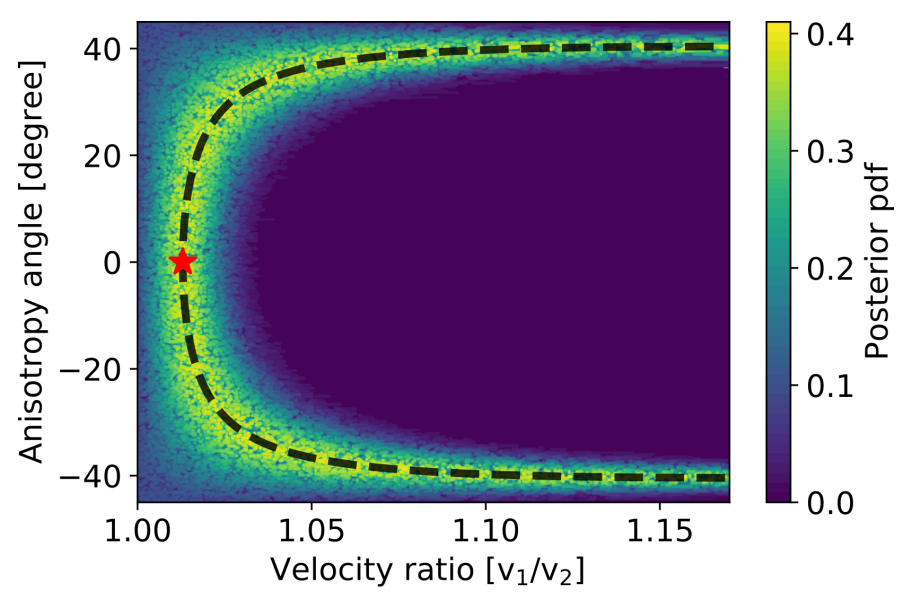

$\star$ True model

- - Predicted non-uniq.

Fig. 4. Posterior probability density function (pdf) related to the unconstrained forward problem in (6). Models with highest pdf correspond to theoretically predicted ones in Fig. 2 (dashed line). They explain equally well traveltimes computed from the true model (red star). For visualization purposes, we display the posterior as a function of the anisotropy angle and the velocity ratio $v_{1} / v_{2}$.

we could reformulate the forward problem to find observations that constrain anisotropic parameters better.

\section{B. Constrained problem}

We illustrate here how the problem can be constrained by combining data from multiple reflector inclinations. We consider the same true model as in the previous example and 32 sources equidistantly distributed along the probe. Now, our artificial observables are $2 \times 32$ traveltime datasets obtained with reflector inclination angles $\alpha=0^{\circ}$ and $\alpha=5^{\circ}$ using (12). Fig. 5 shows the posterior pdf for this case, which has a clear unique maximum that approximately matches the true model location. Unlike the previous example, now traveltimes are determined by a unique set of model parameters. We can quantify uncertainties in the solution using marginal pdfs for each model parameter, shown in Fig. 6. Although the problem is nonlinear, the posterior pdf approximates a multivariate Gaussian distribution. We thus express the solution using the mean and standard deviation of the Gaussian fit of the marginals. Mean values accurately predict true model parameters with standard deviations less than $2.27 \mathrm{~m} / \mathrm{s}$ for velocities and $0.66^{\circ}$ for the anisotropy angle. We also observe that $v_{1}$ is less constrained than $v_{2}$. This is caused by the small aperture of the probe. Ray paths are closer to the direction of $v_{2}$ than $v_{1}$, and we therefore expect larger uncertainties in $v_{1}$. The following examples investigate the impact of reflectorinclination and modelling errors in the solution.

\section{Uncertain reflector inclination}

The Bayesian framework can flexibly incorporate uncertainties about the experimental setup. Suppose we use Bmode images to measure the reflector inclination angle. Such

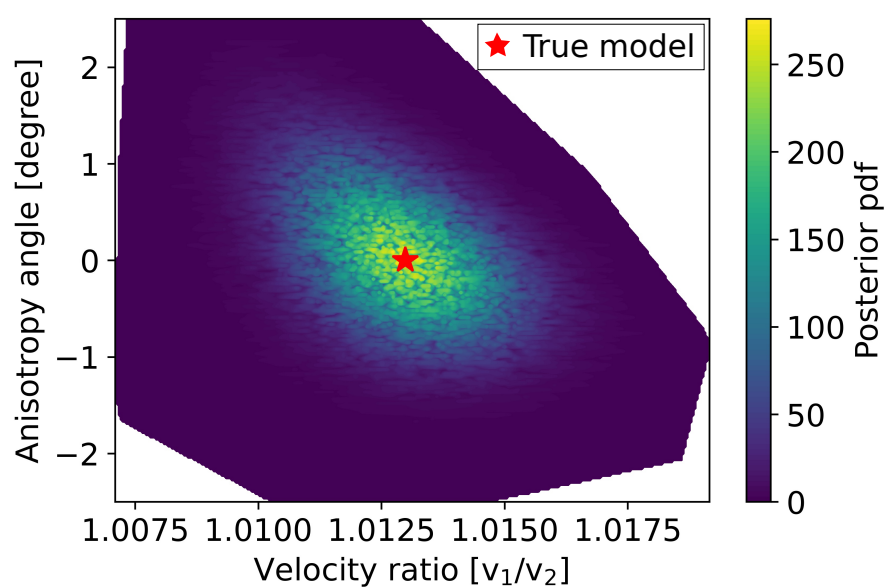

Fig. 5. Posterior probability density function (pdf) when traveltimes from two different reflector inclinations $\left(0^{\circ}\right.$ and $\left.5^{\circ}\right)$ are considered. We use the same true model (red star) as in Fig. 4 and 32 sources equidistantly located. Unsampled models by the algorithm are shown as white areas. The posterior has a unique maximum indicating that model parameters are well constrained by the traveltimes.

measurements will certainly include errors that can propagate into our solution if they are not properly identified. In this context, we suggest taking inclination angles as unknown model parameters, that is, $\mathbf{m}=\left(v_{1}, v_{2}, \varphi, \alpha_{1}, \alpha_{2}\right)$. Then, we can include the information extracted from B-mode images in our prior pdf.

Here we extend the previous example and assume Gaussian priors for $\alpha_{1}$ and $\alpha_{2}$. Because we are interested in understanding how robust the method is to uncertainties in inclination angles, we take the mean of Gaussian priors at $5^{\circ}$ and $10^{\circ}$ with $3^{\circ}$ standard deviation. That is, we shift Gaussian means by $5^{\circ}$ from true values. In this case, the posterior is difficult to visualize due to the dimension of the model space. Fig. 7 displays marginal pdfs for the five model parameters. Again, marginals are approximately Gaussian; therefore, we represent the solution using the mean and standard deviation of their Gaussian fits. The results demonstrate that the anisotropy estimation is robust against uncertainties in reflector inclinations. The most sensitive parameters are $v_{2}$ and $\varphi$ for which standard deviations are two times larger than those in our previous example. Furthermore, the posterior provides accurate values for $\alpha_{1}$ and $\alpha_{2}$, despite the substantial deviations between their prior means and true values. This indicates that the data likelihood is sufficiently informative about reflector inclination angles.

\section{Systematic measurement noise}

This example simulates a situation closer to real applications, with measurement noise and uncertain reflector inclinations. We consider the same problem as before but compute traveltime data from numerical wave propagation simulations using sources with a center frequency of $1 \mathrm{MHz}$. Therefore, our artificial observations include finite-frequency effects that are not accounted for in the forward modelling [see Fig. 3(b)]. 

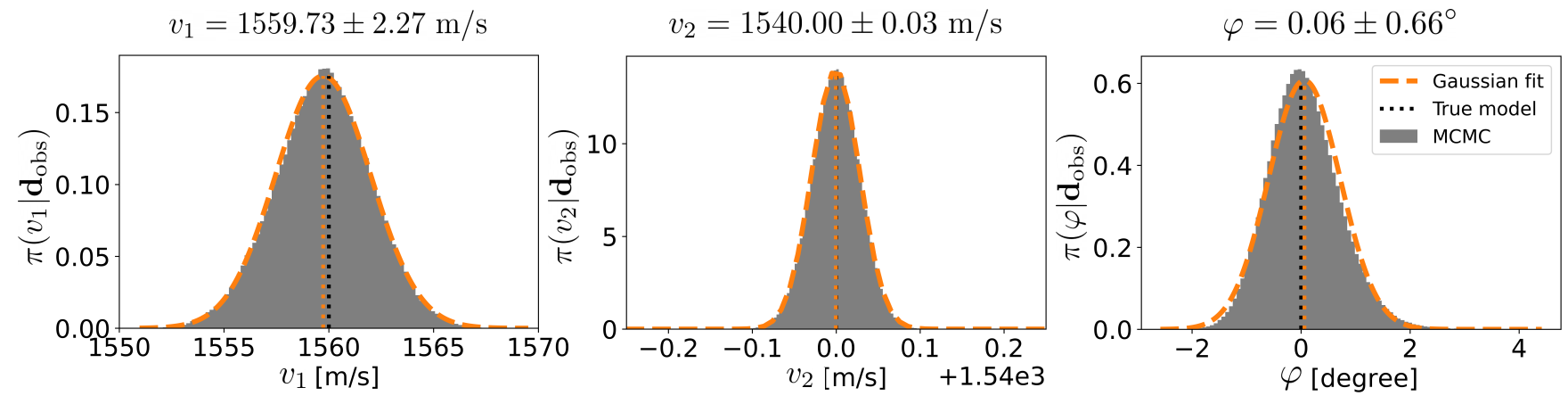

Fig. 6. Marginal probability density functions for $v_{1}, v_{2}$, and $\varphi$. The marginals are histograms obtained with the Markov chain Monte Carlo (MCMC) algorithm and represent the sampling frequency of the values for each model parameter. The Gaussian fit and its mean are indicated with orange dashed lines, and the true model parameters are shown in black. The solution for each parameter is given in terms of the mean and standard deviation of the Gaussian fit, shown on top of the histograms. The velocity across fibers $\left(v_{2}\right)$ is better constrained than the velocity parallel to fibers $\left(v_{1}\right)$.

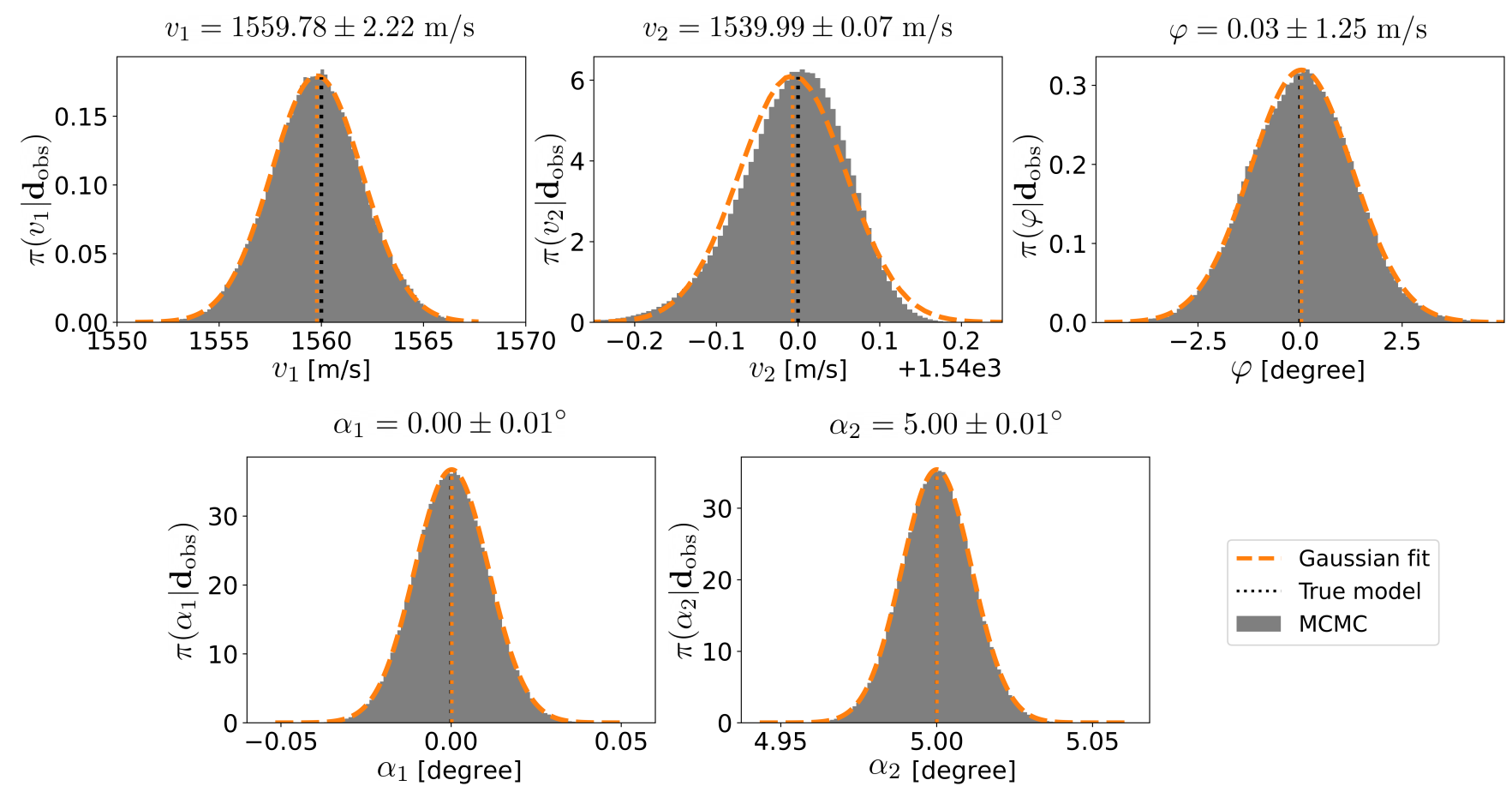

Fig. 7. Marginal probability density functions when reflection inclinations angles $\alpha_{1}$ and $\alpha_{2}$ are unknown model parameters. Inclination angles have Gaussian priors with their mean shifted $5^{\circ}$ from true values and $3^{\circ}$ standard deviation. The solution for each parameter is given in terms of the mean and standard deviation of the Gaussian fit, shown on top of the histograms. Compared to Fig. 6, standard deviations of $v_{2}$ and $\varphi$ increase with uncertain inclination angles. MCMC: Markov chain Monte Carlo.

This can be seen as systematic measurement noise. To make wave propagation simulations computationally affordable, we reduce the probe-reflector distance to $L=6 \mathrm{~cm}$. Because this also reduces traveltimes, we increase the standard deviation of the noise to be consistent with previous examples. Furthermore, to make the example more general, we consider an anisotropy angle of $5^{\circ}$, i.e., the true model is now $\mathbf{m}=\left(1560 \mathrm{~m} / \mathrm{s}, 1540 \mathrm{~m} / \mathrm{s}, 5^{\circ}, 0^{\circ}, 5^{\circ}\right)$.

The marginal pdf of $v_{2}$ deviates most from the Gaussian distribution shown by the rest of model parameters in Fig. 8 . Yet, as a first approximation, we continue using the Gaussian fit for uncertainty quantification. Despite the systematic noise, mean values accurately predict true velocities, meaning that velocity estimates are robust against measurement noise. The anisotropy angle shows the highest sensitivity to noise, with deviations from the true value reaching $\sim 18 \%$. Systematic noise can be seen as modelling errors; thus, deviations between true and maximum a posteriori models are expected. This result demonstrates that a solution given only by the mean, without quantifying uncertainties, is incomplete. All true parameters are predicted within the $95 \%$ confidence interval of the marginals.

Compared to our previous example, standard deviations increase substantially for $v_{2}$ and $\varphi$ and decrease for $v_{1}$. With 

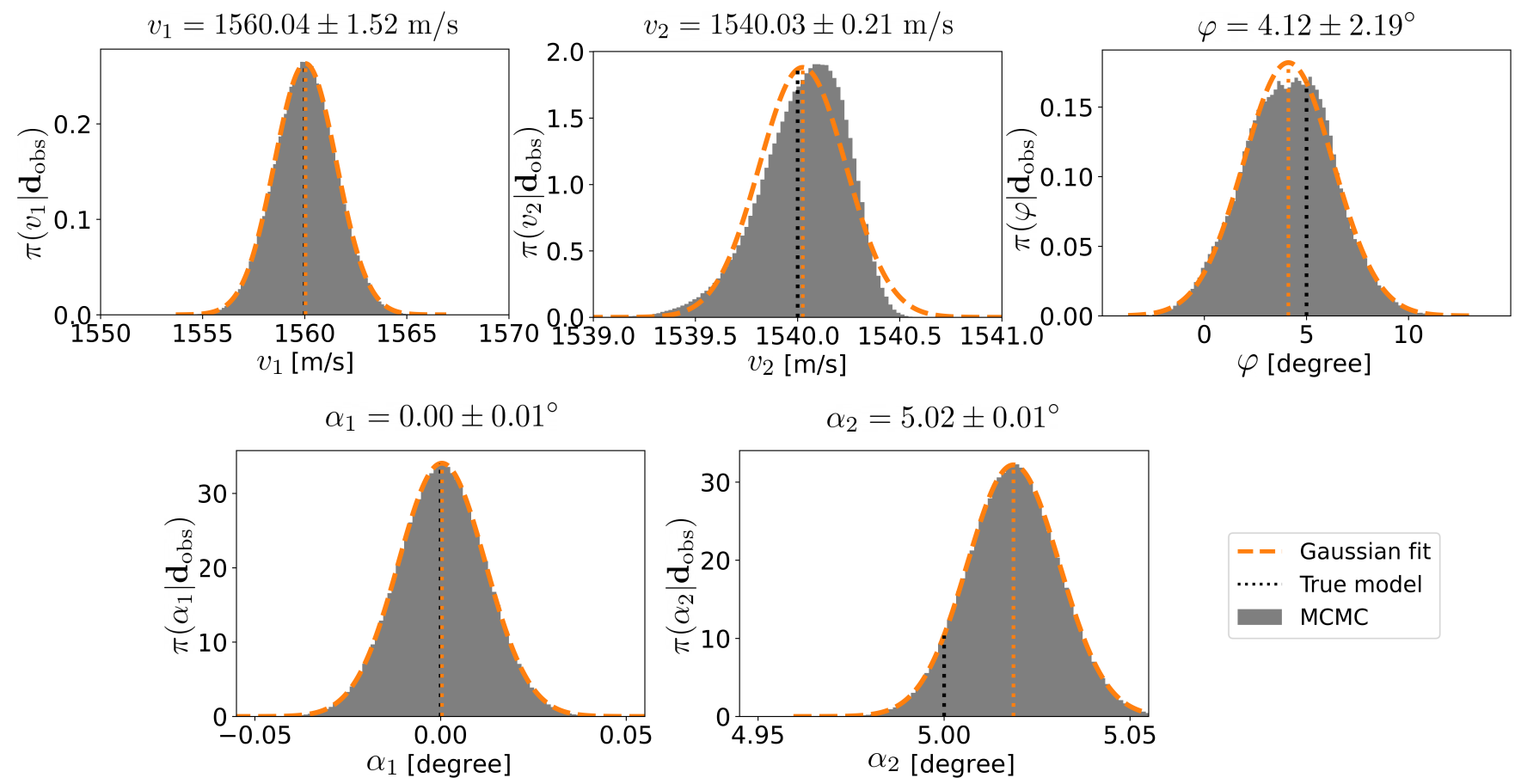

Fig. 8. Marginal probability density functions when artificial observations include systematic noise and reflection inclination angles are uncertain. To better visualize differences between mean and true parameter values, we locate the center of x-axes at true values. The solution for each parameter is given in terms of the mean and standard deviation of the Gaussian fit, shown on top of the histograms. Despite the systematic measurement noise, differences between mean and true values are very small, with the anisotropy angle showing the largest deviations. All true values are predicted within the 95\% confidence interval of the marginals. MCMC: Markov chain Monte Carlo.

tilted anisotropy and reduced probe-reflector distance, ray paths become more parallel to $v_{1}$, constraining the parameter better. The opposite is also true for $v_{2}$, explaining its increased variance. The standard deviation of $\varphi$ appears to be correlated with errors in $v_{2}$, as previously observed. The next subsection analyses these effects more in detail.

\section{E. Impact of experimental setup and medium properties on parameter uncertainties}

Here we analyze changes in parameter uncertainties with varying probe-reflector distance $L$, true anisotropy angle $\varphi$, and velocity differences $\Delta v=v_{1}-v_{2}$. We study these three conditions separately by considering the reference model $\mathbf{m}_{\text {true }}=\left(1560 \mathrm{~m} / \mathrm{s}, 1540 \mathrm{~m} / \mathrm{s}, 5^{\circ}\right)$ and distance $L=10$ $\mathrm{cm}$. We do not consider systematic noise for this analysis. Fig. 9 compares variations in standard deviations of model parameters with varying $L \in[4.8,10] \mathrm{cm}, \varphi \in[0,40]^{\circ}$, and $\Delta v \in[10,30] \mathrm{m} / \mathrm{s}$. These results confirm what we previously observed. For larger anisotropy angles and shorter probereflector distances, standard deviations increase for $v_{2}$ and $\varphi$ while it decreases for $v_{1}$. Velocity differences do not affect $v_{1}$ and $v_{2}$, but $\varphi$ becomes less constrained when these are small. Our forward formulation in (12) shows that traveltimes become independent of $\varphi$ when the medium is isotropic. Therefore, we expect larger uncertainties in $\varphi$ when approaching isotropic conditions. The symmetry of the elliptical anisotropy produces strong correlations between parameters in our results. For instance, when the anisotropy angle is $45^{\circ}$, both velocities are equally constrained. Interestingly, standard deviations of reflector inclination angles remain constant, suggesting that they are nearly uncorrelated to other parameters. In general, we observe that the method is capable of accurately distinguishing velocity differences larger than $2.5 \mathrm{~m} / \mathrm{s}$. This is substantially smaller than longitudinal-wave velocity differences reported in the literature $(>10 \mathrm{~m} / \mathrm{s})$ [14]-[17]. Note that uncertainties could potentially be further reduced by including more sources. Thus, the method presented here is capable of providing accurate and statistically meaningful muscle anisotropy estimates in future clinical applications.

\section{DISCUSSION AND CONCLUSIONS}

We present a novel method to estimate the anisotropy of longitudinal ultrasound waves in transversely isotropic tissue. Until now, only shear waves have been used to characterize tissue anisotropy in clinical applications [22], [23], [25], [26], [30], [45], [46]. However, shear and longitudinal waves interrogate fundamentally different mechanical tissue properties [28]. Their propagation velocities differ by three orders of magnitude, resulting in decoupled relationships between the two velocities and elastic moduli [31]. Hence, our work not only complements other studies on the topic but is pivotal to characterize mechanical tissue properties comprehensively.

\section{A. Experimental setup}

Our method relies on an experimental setup with a reflector parallel to the linear ultrasound probe, with tissue in 
(a)

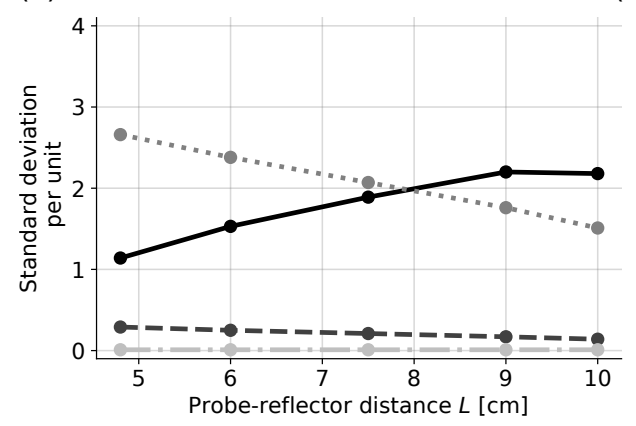

(b)

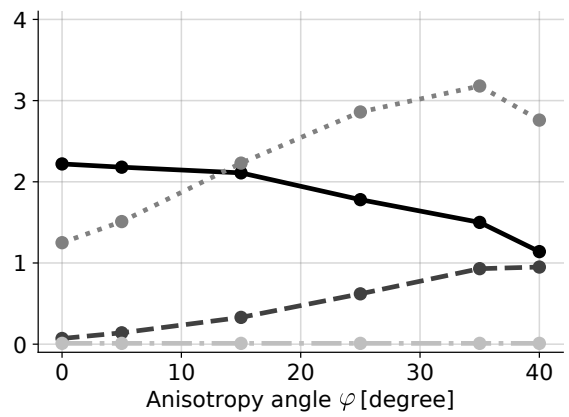

(c)

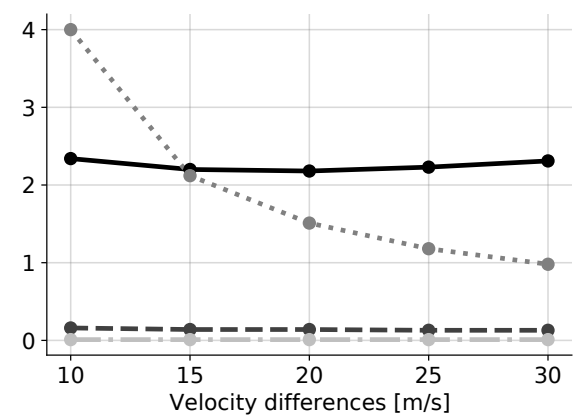

Fig. 9. Standard deviations of model parameters as a function of experimental setup and medium properties. The reference model and experimental parameters are $\mathbf{m}_{\text {true }}=\left(1560 \mathrm{~m} / \mathrm{s}, 1540 \mathrm{~m} / \mathrm{s}, 5^{\circ}\right)$ and $L=10 \mathrm{~cm}$, respectively. We modify (a) the probe-reflector distance $L$ from $4.8 \mathrm{~cm}$ to $10 \mathrm{~cm}$, (b) true anisotropy angle $\varphi$ from $0^{\circ}$ to $40^{\circ}$, and true velocity differences $\Delta v=v_{1}-v_{2}$ from $10 \mathrm{~m} / \mathrm{s}$ to $30 \mathrm{~m} / \mathrm{s}$. The estimated anisotropy angle becomes more uncertain when $L$ decreases, the true $\varphi$ increases, or the medium approaches isotropy. In general, we can distinguish velocity differences larger than $2.5 \mathrm{~m} / \mathrm{s}$.

between. While this setup has already been applied in various clinical studies [12], [13], [31], [33]-[35], it differs from those suggested for shear-wave anisotropy estimation, which requires either 2D matrix-array probes [23], [25], [46] or the rotation of linear probes [22], [26], [30], [45]. The difference in setups is a direct consequence of differences in shearand longitudinal-wave propagation directions. Shear waves generated using elastography techniques are approximately plane waves propagating in the lateral direction. In such a case, velocities at different propagation directions can only be assessed if the probe is rotated. Longitudinal waves, on the other hand, propagate in the axial direction perpendicular to the probe. By including a reflector, we generate ray paths with components also in the lateral direction. This allows us to interrogate different propagation directions, similar to the probe rotation in shear-wave techniques. In any case, both shear- and longitudinal-wave anisotropy quantification require adapting experimental setups currently used in clinical practice.

\section{B. Assumptions}

The forward problem derived here rests on several assumptions. We approximate muscles as transversely isotropic, and more specifically, we assume elliptical anisotropy. This assumption substantially simplifies our analytical derivations and is in line with other works [45]-[47]. While the shearwave velocity does exhibit elliptical dependence in transversely isotropic media (at least for waves polarized in the isotropy plane), wavefronts of longitudinal waves are not generally ellipsoidal. However, we demonstrate in Appendix A that elliptical anisotropy does occur if elastic moduli satisfy $c_{12}=\sqrt{c_{11} c_{22}}$. This condition has been empirically validated in muscles in ex-vivo animal studies [29], [47]. The elliptical anisotropy is therefore a reasonable assumption for this study. In addition, our forward problem also assumes the principal fiber direction in the $x_{1} x_{2}$-plane. This can be controlled using B-mode images and should be ensured for a meaningful interpretation of the results.

\section{Optimization}

Traveltimes and anisotropy parameters are nonlinearly related; accordingly, we solve the inverse problem using Bayesian inference. Compared to gradient-based optimization techniques, our choice is computationally more demanding and may not suit clinical time constraints. However, our goal was to analyze the nature of the proposed problem for which uncertainty quantification is indispensable. For this task, Bayesian inference is a more powerful approach. For example, this analysis was useful to demonstrate that anisotropy is accurately constrained when traveltimes from different reflector inclinations are combined. An inclination in the reflector is unavoidable in practice and generally regarded as a source of unwanted noise. Here we have resignified its value and transformed it into a key ingredient for successfully solving the problem.

Ideally, we would like to accelerate the inversion and maintain the ability to quantify uncertainties, which may be crucial for clinical decision-making. In the current implementation, we sample the posterior using the Metropolis-Hastings algorithm, which evaluates approximately $10^{5}$ models per minute on a single CPU from a laptop computer with 15$20 \%$ acceptance rate. This algorithm is known to have a poor acceptance rate, meaning that a large number of samples is needed to approximate the posterior sufficiently well [48]. The performance can be significantly improved incorporating, for instance, information from derivatives of the log posterior in order to guide the sampler towards high-probability regions of the model space. Hamiltonian Monte Carlo was designed to tackle this [48], [49] and is well suited for our problem, where analytical expressions for derivatives are available. Alternatively, due to the nearly Gaussian structure of the posterior, we could approximate it locally using second derivatives of the forward problem around the solution found by gradientbased optimization techniques [50]. Future research will focus on exploring these options. 


\section{Expected uncertainties}

For nonlinear problems, the posterior pdf depends on the anisotropy model. Still, we can draw some general conclusions about uncertainties in inferred anisotropy parameters: (1) Velocities in directions that are more parallel to the probe (i.e., fiber direction) are generally less constrained than those in perpendicular directions due to the small aperture of the acquisition setup. (2) The anisotropy angle $\varphi$ is the least constrained parameter with relative uncertainties that are two orders of magnitudes larger than those for velocities. In fact, $\varphi$ becomes increasingly less reliable as velocity differences approach isotropic conditions. Yet, such uncertainties do not affect velocity estimates, which are robust against noise. (3) In principle, our method is capable of accurately distinguishing velocity differences four times smaller $(2.5 \mathrm{~m} / \mathrm{s})$ than those observed in muscle tissue $(>10 \mathrm{~m} / \mathrm{s})$ [14]-[17]. (4) Overall, the largest standard deviations in $\varphi\left(4^{\circ}\right)$ are substantially smaller than those reported in similar numerical studies with shear waves $\left(5.6^{\circ}-36.3^{\circ}\right)$ [23]. Maximum relative errors in velocities are also considerably lower in our case $(0.2 \%$ versus 20\%) [23]. It suggests that quantifying anisotropy in longitudinal waves could potentially be more robust than in shear waves, which show moreover higher sensitivity to confounding variables than longitudinal waves [31].

\section{E. Clinical interest}

The arrangement of fibers in the muscle causes anisotropy in mechanical tissue properties. Muscles can be seen as a stack of thin, homogeneous layers of different properties. At large scales, such a structure behaves as a homogeneous transversely isotropic medium whose properties are related to the finescale medium through the effective medium theory [51], [52]. Consequently, anisotropy parameters are correlated to both muscle composition and architecture, which are affected by musculoskeletal disorders. For instance, changes in the number and type of fibers will lead to changes in anisotropy parameters. Therefore, quantifying this property with ultrasound could ultimately offer a cost-efficient, multi-parametric biomarker to assess disease-related changes in muscle mass (composition) and function (architecture).

\section{APPENDIX A}

\section{ELLIPTICAL ANISOTROPY}

This appendix discusses the elliptical anisotropy assumption in muscle and shows the conditions under which (1) is satisfied. The wave surface given by (1) is an ellipsoid only if the slowness (reciprocal of the phase velocity) surface is also an ellipsoid [32], [53]. We therefore focus on analyzing the expression for phase velocity.

For simplicity, we consider a two-dimensional transversely isotropic medium with the symmetry axis parallel to $x_{1}$ direction. The elastic stiffness tensor $c_{i j k l}$ characterizing this medium has five independent components, which are $c_{11}$, $c_{12}, c_{22}, c_{44}$, and $c_{66}$ in Voigt notation. The parameters $c_{44}$ and $c_{66}$ are related to shear moduli; thus, in soft tissue, $c_{44}, c_{66} \ll c_{11}, c_{12}, c_{22}$ [29]. We can relate the stiffness tensor to phase velocities $V$ through the Christoffel equation

$$
\operatorname{det}\left[c_{i j k l} n_{j} n_{l}-\rho V^{2} \delta_{i k}\right]=0,
$$

where $\rho$ denotes medium density, the Kronecker delta $\delta_{i j}$ is equal to one when $i=j$ and zero otherwise, and $n_{i}$ refers to the $i$ th component of the wavefront normal vector (slowness vector). For an arbitrary wavefront direction $\mathbf{n}=(\sin \phi, \cos \phi),(19)$ leads to

$$
V^{2}(\phi)=\frac{1}{2 \rho}\left[c_{11} \sin ^{2} \phi+c_{22} \cos ^{2} \phi+G(\phi)\right]
$$

for longitudinal waves, with

$$
G(\phi)=\left[\left(c_{11} \sin ^{2} \phi-c_{22} \cos ^{2} \phi\right)^{2}+c_{12}^{2} \sin ^{2} 2 \phi\right]^{\frac{1}{2}} .
$$

The elliptical anisotropy assumption is only valid when the slowness surface in (20) is an ellipse, which is generally not the case. Only when the medium satisfies $c_{12}=\sqrt{c_{11} c_{22}}$, (20) reduces to the ellipse

$$
V^{2}(\phi)=\frac{1}{\rho}\left[c_{11} \sin ^{2} \phi+c_{22} \cos ^{2} \phi\right],
$$

with semi-axes $\sqrt{\rho / c_{11}}$ and $\sqrt{\rho / c_{22}}$. In muscle tissue, empirical studies have shown that $c_{12} \approx \sqrt{c_{11} c_{22}}$ [29], [47], with reported deviations that are below $0.3 \%$. This justifies the elliptical anisotropy model used in this study.

\section{ACKNOWLEDGMENT}

The authors would like to thank Sergio Sanabria for initial discussions and Christian Boehm for helping with numerical wave propagation simulations. We also thank Andreas Fichtner for supporting us with computing time in the Swiss National Supercomputing Center (CSCS project s1040). The Python code used for generating the inversion results of this paper can be downloaded from GitHub (https://github.com/naiarako/UltrasoundAnisotropy) in the form of a self-explanatory Jupyter Notebook.

\section{REFERENCES}

[1] E. A. O'Flynn, J. Fromageau, A. E. Ledger, A. Messa, A. D'Aquino, M. J. Schoemaker, M. Schmidt, N. Duric, A. J. Swerdlow, and J. C. Bamber, "Ultrasound tomography evaluation of breast density: A comparison with noncontrast magnetic resonance imaging," Investigative Radiology, vol. 52, no. 6, pp. 343-348, 2017.

[2] H. Gemmeke, T. Hopp, M. Zapf, C. Kaiser, and N. V. Ruiter, "3D ultrasound computer tomography: Hardware setup, reconstruction methods and first clinical results," Nuclear Instruments and Methods in Physics Research Section A: Accelerators, Spectrometers, Detectors and Associated Equipment, vol. 873, pp. 59-65, 2017, imaging 2016.

[3] M. Jakovljevic, S. Hsieh, R. Ali, G. Chau Loo Kung, D. Hyun, and J. J. Dahl, "Local speed of sound estimation in tissue using pulseecho ultrasound: Model-based approach," The Journal of the Acoustical Society of America, vol. 144, no. 1, pp. 254-266, 2018.

[4] S. J. Sanabria, M. B. Rominger, and O. Goksel, "Speed-of-sound imaging based on reflector delineation," IEEE Transactions on Biomedical Engineering, vol. 66, no. 7, pp. 1949-1962, 2019.

[5] J. Wiskin, B. Malik, D. Borup, N. Pirshafiey, and J. Klock, "Full wave $3 \mathrm{D}$ inverse scattering transmission ultrasound tomography in the presence of high contrast," Scientific Reports, vol. 10, no. 1, p. 20166, 2020. 
[6] N. Korta Martiartu, C. Boehm, and A. Fichtner, "3-D Wave-EquationBased Finite-Frequency Tomography for Ultrasound Computed Tomography," IEEE Transactions on Ultrasonics, Ferroelectrics, and Frequency Control, vol. 67, no. 7, pp. 1332-1343, 2020.

[7] P. Stähli, M. Frenz, and M. Jaeger, "Bayesian approach for a robust speed-of-sound reconstruction using pulse-echo ultrasound," IEEE Transactions on Medical Imaging, vol. 40, no. 2, pp. 457-467, 2021.

[8] G. Zografos, D. Koulocheri, P. Liakou, M. Sofras, S. Hadjiagapis, M. Orme, and V. Marmarelis, "Novel technology of multimodal ultrasound tomography detects breast lesions," European Radiology, vol. 23, no. 3, pp. 673-683, 2013

[9] L. Ruby, S. J. Sanabria, K. Martini, K. J. Dedes, D. Vorburger, E. Oezkan, T. Frauenfelder, O. Goksel, and M. B. Rominger, "Breast cancer assessment with pulse-echo speed of sound ultrasound from intrinsic tissue reflections: Proof-of-concept," Investigative Radiology, vol. 54, no. 7, pp. 419-427, 2019.

[10] M. Dioguardi Burgio, M. Imbault, M. Ronot, A. Faccinetto, B. E. Van Beers, P.-E. Rautou, L. Castera, J.-L. Gennisson, M. Tanter, and V. Vilgrain, "Ultrasonic adaptive sound speed estimation for the diagnosis and quantification of hepatic steatosis: A pilot study," Ultraschall Med, vol. 40, no. 6, pp. 722-733, 2019.

[11] M. Imbault, A. Faccinetto, B.-F. Osmanski, A. Tissier, T. Deffieux, J.-L. Gennisson, V. Vilgrain, and M. Tanter, "Robust sound speed estimation for ultrasound-based hepatic steatosis assessment," Physics in Medicine and Biology, vol. 62, no. 9, pp. 3582-3598, apr 2017. [Online]. Available: https://doi.org/10.1088/1361-6560/aa6226

[12] S. J. Sanabria, K. Martini, G. Freystätter, L. Ruby, O. Goksel, T. Frauenfelder, and M. B. Rominger, "Speed of sound ultrasound: a pilot study on a novel technique to identify sarcopenia in seniors," European Radiology, vol. 29, no. 1, pp. 3-12, 2019.

[13] L. Ruby, A. Kunut, D. N. Nakhostin, F. A. Huber, T. Finkenstaedt, T. Frauenfelder, S. J. Sanabria, and M. B. Rominger, "Speed of sound ultrasound: comparison with proton density fat fraction assessed with Dixon MRI for fat content quantification of the lower extremity," European Radiology, vol. 30, no. 10, p. 5272, 2020.

[14] C. R. Mol and P. A. Breddels, "Ultrasound velocity in muscle," The Journal of the Acoustical Society of America, vol. 71, no. 2, pp. 455461, 1982.

[15] D. E. Goldman and J. R. Richards, "Measurement of high-frequency sound velocity in mammalian soft tissues," The Journal of the Acoustical Society of America, vol. 26, no. 6, pp. 981-983, 1954.

[16] W. O'Brien and J. Olerud, "Ultrasonic assessment of tissue anisotropy," in 1995 IEEE Ultrasonics Symposium. Proceedings. An International Symposium, vol. 2, 1995, pp. 1145-1148 vol.2.

[17] E. D. Verdonk, S. A. Wickline, and J. G. Miller, "Anisotropy of ultrasonic velocity and elastic properties in normal human myocardium," The Journal of the Acoustical Society of America, vol. 92, no. 6, pp. 3039-3050, 1992.

[18] K. A. Topp and W. D. O’Brien, "Anisotropy of ultrasonic propagation and scattering properties in fresh rat skeletal muscle in vitro," The Journal of the Acoustical Society of America, vol. 107, no. 2, pp. 1027 1033, 2000.

[19] B. H. Goodpaster, S. W. Park, T. B. Harris, S. B. Kritchevsky, M. Nevitt, A. V. Schwartz, E. M. Simonsick, F. A. Tylavsky, M. Visser, and f. t. H A. S. Newman, Anne B., "The Loss of Skeletal Muscle Strength, Mass, and Quality in Older Adults: The Health, Aging and Body Composition Study," The Journals of Gerontology: Series A, vol. 61, no. 10, pp. 1059-1064, 102006.

[20] A. J. Cruz-Jentoft, G. Bahat, J. Bauer, Y. Boirie, O. Bruyère, T. Cederholm, C. Cooper, F. Landi, Y. Rolland, A. A. Sayer, S. M. Schneider, C. C. Sieber, E. Topinkova, M. Vandewoude, M. Visser, M. Zamboni, W. G. for the European Working Group on Sarcopenia in Older People 2 (EWGSOP2), and the Extended Group for EWGSOP2, "Sarcopenia: revised European consensus on definition and diagnosis," Age and Ageing, vol. 48, no. 1, pp. 16-31, 092018.

[21] M. V. Narici, C. N. Maganaris, N. D. Reeves, and P. Capodaglio, "Effect of aging on human muscle architecture," Journal of Applied Physiology, vol. 95, no. 6, pp. 2229-2234, 2003, pMID: 12844499.

[22] W.-N. Lee, M. Pernot, M. Couade, E. Messas, P. Bruneval, A. Bel, A. A. Hagege, M. Fink, and M. Tanter, "Mapping myocardial fiber orientation using echocardiography-based shear wave imaging," IEEE Transactions on Medical Imaging, vol. 31, no. 3, pp. 554-562, 2012.

[23] M. Correia, T. Deffieux, S. Chatelin, J. Provost, M. Tanter, and M. Pernot, "3D elastic tensor imaging in weakly transversely isotropic soft tissues," Physics in Medicine \& Biology, vol. 63, no. 15, p. 155005, jul 2018.

[24] W.-N. Lee, B. Larrat, M. Pernot, and M. Tanter, "Ultrasound elastic tensor imaging: comparison with MR diffusion tensor imaging in the myocardium," Physics in Medicine and Biology, vol. 57, no. 16, pp. 5075-5095, jul 2012. [Online]. Available: https://doi.org/10.1088/0031$9155 / 57 / 16 / 5075$

[25] M. Wang, B. Byram, M. Palmeri, N. Rouze, and K. Nightingale, "Imaging transverse isotropic properties of muscle by monitoring acoustic radiation force induced shear waves using a 2-D matrix ultrasound array," IEEE Transactions on Medical Imaging, vol. 32, no. 9, pp. 16711684, 2013.

[26] M. Hossain, C. J. Moore, and C. M. Gallippi, "Acoustic radiation force impulse-induced peak displacements reflect degree of anisotropy in transversely isotropic elastic materials," IEEE Transactions on Ultrasonics, Ferroelectrics, and Frequency Control, vol. 64, no. 6, pp. 989-1001, 2017.

[27] M. M. Hossain, R. K. Detwiler, E. H. Chang, M. C. Caughey, M. W. Fisher, T. C. Nichols, E. P. Merricks, R. A. Raymer, M. Whitford, D. A. Bellinger, L. E. Wimsey, and C. M. Gallippi, "Mechanical anisotropy assessment in kidney cortex using arfi peak displacement: Preclinical validation and pilot in vivo clinical results in kidney allografts," IEEE Transactions on Ultrasonics, Ferroelectrics, and Frequency Control, vol. 66, no. 3, pp. 551-562, 2019.

[28] T. Glozman and H. Azhari, "A method for characterization of tissue elastic properties combining ultrasonic computed tomography with elastography," Journal of Ultrasound in Medicine, vol. 29, no. 3, pp. 387398,2010 .

[29] S. F. Levinson, "Ultrasound propagation in anisotropic soft tissues: The application of linear elastic theory," Journal of Biomechanics, vol. 20, no. 3, pp. 251-260, 1987 .

[30] J.-L. Gennisson, T. Deffieux, E. Macé, G. Montaldo, M. Fink, and M. Tanter, "Viscoelastic and anisotropic mechanical properties of in vivo muscle tissue assessed by supersonic shear imaging," Ultrasound in Medicine \& Biology, vol. 36, no. 5, pp. 789-801, 2010.

[31] N. Korta Martiartu, D. Nakhostin, L. Ruby, T. Frauenfelder, M. B. Rominger, and S. J. Sanabria, "Speed of sound and shear wave speed for calf soft tissue composition and nonlinearity assessment," Quantitative Imaging in Medicine and Surgery, vol. 11, no. 9, 2021.

[32] B. S. Byun, "Seismic parameters for media with elliptical velocity dependencies," GEOPHYSICS, vol. 47, no. 12, pp. 1621-1626, 1982.

[33] S. J. Sanabria, O. Goksel, K. Martini, S. Forte, T. Frauenfelder, R. A. Kubik-Huch, and M. B. Rominger, "Breast-density assessment with hand-held ultrasound: A novel biomarker to assess breast cancer risk and to tailor screening?" European Radiology, vol. 28, no. 8, pp. 31653175,2018

[34] L. Ruby, S. J. Sanabria, A. S. Obrist, K. Martini, S. Forte, O. Goksel, T. Frauenfelder, R. A. Kubik-Huch, and M. B. Rominger, "Breast density assessment in young women with ultrasound based on speed of sound: Influence of the menstrual cycle," Medicine, vol. 98, no. 25, p. e16123, 2019.

[35] L. Ruby, S. J. Sanabria, K. Martini, T. Frauenfelder, G. N. Jukema, O. Goksel, and M. B. Rominger, "Quantification of immobilizationinduced changes in human calf muscle using speed-of-sound ultrasound: An observational pilot study," Medicine, vol. 100, no. 10, p. e23576, 2021.

[36] M. Afanasiev, C. Boehm, and et al., "Modular and flexible spectralelement waveform modelling in two and three dimensions," Geophysical Journal International, vol. 216, no. 3, pp. 1675-1692, 112018.

[37] A. Tarantola, Inverse Problem Theory and Methods for Model Parameter Estimation. Society for Industrial and Applied Mathematics, 2005.

[38] K. Mosegaard and A. Tarantola, "Monte carlo sampling of solutions to inverse problems," Journal of Geophysical Research: Solid Earth, vol. 100, no. B7, pp. 12 431-12 447, 1995.

[39] T. Bayes and R. Price, "An essay towards solving a problem in the doctrine of chances," Philosophical Transactions of the Royal Society of London, vol. 53, pp. 370-418, 1763.

[40] A. Tarantola and B. Valetter, "Inverse problems = quest for information," Journal of Geophysics, vol. 50, no. 1, pp. 159-170, 1982.

[41] N. Korta Martiartu, C. Boehm, V. Hapla, H. Maurer, I. J. Balic, and A. Fichtner, "Optimal experimental design for joint reflectiontransmission ultrasound breast imaging: From ray- to wave-based methods," The Journal of the Acoustical Society of America, vol. 146, no. 2, pp. 1252-1264, 2019. 
[42] N. Metropolis, A. W. Rosenbluth, M. N. Rosenbluth, A. H. Teller, and E. Teller, "Equation of state calculations by fast computing machines," The Journal of Chemical Physics, vol. 21, no. 6, pp. 1087-1092, 1953.

[43] W. K. Hastings, "Monte carlo sampling methods using markov chains and their applications," Biometrika, vol. 57, no. 1, pp. 97-109, 1970.

[44] L. Tierney, "Markov Chains for Exploring Posterior Distributions," The Annals of Statistics, vol. 22, no. 4, pp. 1701 - 1728, 1994.

[45] J.-L. Gennisson, S. Catheline, S. Chaffaï, and M. Fink, "Transient elastography in anisotropic medium: Application to the measurement of slow and fast shear wave speeds in muscles," The Journal of the Acoustical Society of America, vol. 114, no. 1, pp. 536-541, 2003.

[46] M. M. Hossain and C. M. Gallippi, "Electronic point spread function rotation using a three-row transducer for arfi-based elastic anisotropy assessment: In silico and experimental demonstration," IEEE Transactions on Ultrasonics, Ferroelectrics, and Frequency Control, vol. 68, no. 3, pp. 632-646, 2021.

[47] D. Royer, J.-L. Gennisson, T. Deffieux, and M. Tanter, "On the elasticity of transverse isotropic soft tissues (1)," The Journal of the Acoustical Society of America, vol. 129, no. 5, pp. 2757-2760, 2011.

[48] M. Betancourt, "A Conceptual Introduction to Hamiltonian Monte Carlo," 2018.

[49] S. Duane, A. Kennedy, B. J. Pendleton, and D. Roweth, "Hybrid monte carlo," Physics Letters B, vol. 195, no. 2, pp. 216-222, 1987.

[50] T. Bui-Thanh, O. Ghattas, J. Martin, and G. Stadler, "A computational framework for infinite-dimensional bayesian inverse problems part i: The linearized case, with application to global seismic inversion," SIAM Journal on Scientific Computing, vol. 35, no. 6, pp. A2494-A2523, 2013.

[51] G. E. Backus, "Long-wave elastic anisotropy produced by horizontal layering," Journal of Geophysical Research (1896-1977), vol. 67, no. 11, pp. 4427-4440, 1962.

[52] T. H. Jordan, "An effective medium theory for three-dimensional elastic heterogeneities," Geophysical Journal International, vol. 203, no. 2, pp. 1343-1354, 102015.

[53] K. Helbig, "Elliptical anisotropy-its significance and meaning," GEOPHYSICS, vol. 48, no. 7, pp. 825-832, 1983. 